\title{
Structural and Electronic Properties of Low-Dimensional C-Nanoassemblies and Possible Analogues for Si (and Ge)
}

\author{
N. H. March ${ }^{1,2,3}$ and A. Rubio ${ }^{4}$ \\ ${ }^{1}$ DIPC, San Sebastian, Donosita-San Sebastian, 20078 Gipuzkoa, Spain \\ ${ }^{2}$ Department of Physics, University of Antwerp, 2000 Antwerpen, Belgium \\ ${ }^{3}$ Oxford University, Oxford, OX 12JD, UK \\ ${ }^{4}$ Nano-Bio Spectroscopy Group and ETSF Scientific Development Centre, Depart Física de Materiales, \\ Universidad del País Vasco, Centro de Física de Materiales CSIC-UPV/EHU-MPC and DIPC, \\ Centro Joxe Mari Korta Avenida de Tolosa, Donostia-San Sebastian, 72, 20018 Gipuzkoa, Spain
}

Correspondence should be addressed to N. H. March, arubio@sc.ehu.es

Received 11 June 2010; Accepted 20 September 2010

Academic Editor: Teng Li

Copyright (C) 2011 N. H. March and A. Rubio. This is an open access article distributed under the Creative Commons Attribution License, which permits unrestricted use, distribution, and reproduction in any medium, provided the original work is properly cited.

The delocalised nature of $\pi$-electrons in carbon-based compounds has opened a huge path for new fundamental and technological developments using carbon-based materials of different dimensionality (from clusters, to surfaces, nanotubes and graphene, among others). The success of this field has prompted the proposal that other inorganic structures based on $\mathrm{B}$ and $\mathrm{N}$ and more recently on Si and Ge could be formed with specific structural, mechanical, and electronic properties. In this paper we provide an analysis of the similarities of the two fields starting from the analysis of the $\mathrm{Si}_{6} \mathrm{H}_{6}$ molecule, the analogue of the benzene molecule but now being nonplanar. Then we move to the study of the two-dimensional (buckled) analogues of graphene but now formed by Si and Ge. Similarly, we look to nonplanar compounds based on boron and boron-carbon nitrogen composites. In particular, we focus on the mechanical properties of those new materials that exhibit a very high stiffness, resilience, and flexibility. Possible applications in the fields of catalysis, lubrication, electronic, and photonic devices now seem a likely by-product. We also address future directions triggered by the predicted superconducting properties of graphene, among other areas.

\section{Background and Outline}

Both authors have been concerned since their early researches with (somewhat different) aspects of $\mathrm{C}$ nanoassemblies. Thus in Section 1.1 below, work on the electron density in $\mathrm{C}_{6} \mathrm{H}_{6}$ is briefly summarized while in Section 3 studies on mechanical properties of $\mathrm{C}$ nanotubes are considered. Thus, structural, mechanical, and electronic properties of such $\mathrm{C}$ assemblies will be reviewed below, drawing of course on the contributions of many authors, concerned with both theory and experiment. With the background also of the present authors on $\mathrm{B}, \mathrm{N}$, and $\mathrm{Si}$ being relevant again to nanoassemblies, it seemed natural from time to time to refer to these atoms (plus Ge also occasionally) in the course of present discussion, to compare and contrast with purely Cbased structures.
1.1. Electron Density in Benzene, Using Experimentally Known Geometry. In early work [1], one of us compared the ground-state electron density of benzene at its experimentally known geometry as given by the LCAO-MO approximation with that from the semiclassical Thomas-Fermi theory [2], the forerunner of modern density functional theory [3]. Some further contact with experiment came from comparison of the calculated electron densities with available $\mathrm{X}$-ray diffraction experiments of Robertson et al. $[4,5]$ on naphthalene, in its crystalline state.

As will be discussed in somewhat more detail in Section 3, it was considerably later that Grassi et al. [6] carried out first-principles calculations on $\mathrm{Si}_{6} \mathrm{H}_{6}$, using both restricted and unrestricted Hartree-Fock (HF) theory. While their main objective was to examine theoretically, as chemical bonds were stretched, whether there would be 
a Coulson-Fischer-like point (for $\mathrm{H}_{2}$ in their example [7]) in the sense of a transition from ring currents to localized $\pi$ electrons, in [6] a buckled $\mathrm{Si}_{6} \mathrm{H}_{6}$ structure was predicted, to obtain all real vibrational frequencies.

In connection with graphene, in a work on exfoliated monolayer $\mathrm{MnO}_{2}$ sheets, Wang et al. [8] wrote, and we quote them: "strictly speaking no perfect $2 \mathrm{D}$ crystalline sheet structure would exist in free space unless it became an inherent part of a bulk crystal".

In the above context, Meyer et al. [9], and also Stankovich et al. [10], found significant local curvature within graphene. In relation to the above remarks on the planar benzene molecule and, as will be discussed a little more in connection with the distortion from planarity of the as yet unsynthesized molecule $\mathrm{Si}_{6} \mathrm{H}_{6}$, we can expect any $\mathrm{Si}$ or $\mathrm{Ge}$ analogues of graphene which may be synthesized in the future to have notable departures from planarity. These will, of course, affect molecular vibrational properties and hence mechanical behaviour.

Returning briefly to benzene [1-5], a quite recent survey of ab initio calculations can be found in Moran et al. [11].

\subsection{Strained Graphenic Fragments due to Embedded Impurity} Cluster. Dietz et al. [12] considered a variety of localized defect states (e.g., impurity defects) as well as topological defects, in two-dimensional graphite. Independently, Peeters et al. [13] have reported results on a planar $B_{12}$ cluster embedded in graphenic fragments of different sizes. Below as we are dominantly concerned with nanomaterials, we focus on the main findings of Peeters et al. The basic motivation for their study was twofold: first, to shed light on bonding in a variety of carborazines (systems involving $\mathrm{C}, \mathrm{B}$ and $\mathrm{N}$ atoms), either in pairs $[14,15]$ or with all three different types of atoms present, and second to gain understanding of the way the $\mathrm{C}$ nuclei relax in such two-dimensional fragments due to the stress induced when a defect structure replaces a portion of the network, so that perfect accommodation cannot be retained.

Within the first octet row of the Periodic Table where directed bonding is particularly in evidence, $\mathrm{B}, \mathrm{C}$, and $\mathrm{N}$ are the most highly branched nonmetal species, which are then natural combinations to build up complex networks. Further understanding of bonding involving $\mathrm{C}$ with $\mathrm{B}$ and $\mathrm{N}$ holds promise of important consequences for nanoscale structures and in design of materials. In addition, clusters embedded in, as well as adsorbed on, large polycyclic aromatic hydrocarbons have signicance for molecular electronics (see, e.g., Stabel et al. [16]).

The $B_{12}$ clusters discussed below are favorable for chemical bonding in free space and have been studied by Boustani (see e.g., $[17,18]$ ). Below, we report results from Peeters et al. [13] in which two of Boustani's more favorable $\mathrm{B}_{12}$ clusters are considered, surrounded first by quite a small number of $\mathrm{C}$ atoms. The chemical picture for such small carborane clusters was then refined by Hartree-Fock calculations in order to find (i) the stable cluster geometry and (ii) the deformation electron density distribution. Then, we summarize the effects of adding on the periphery of such nanofragments, further $\mathrm{C}$ atoms, all the surface $\mathrm{C}$ species being bonded to peripheral $\mathrm{H}$ atoms.

Turning to specific B clusters and fragments, Figure 1(a) shows one $B_{12}$ cluster to be embedded in a series of graphenic fragments. A second $B_{12}$ cluster studied by Boustani and others is depicted in part (b) of Figure 1 and this is also treated briefly below.

A good choice for an appropriate graphenic fragment is the 16-atom "pyrene" cluster. In the small cluster studied by Peeters et al. [13], the $\mathrm{B}_{12}$ clusters were surrounded by just two layers of $\mathrm{C}$ atoms. Then $\mathrm{H}$ atoms, as mentioned above, are added at the periphery of these fragments. The formula for these smallest graphenic fragments is $\mathrm{B}_{12} \mathrm{C}_{64} \mathrm{H}_{22}$. As discussed by Peeters et al., the geometry of $\mathrm{B}_{12}$ as in Figure 1(a) is significantly different from the geometry of an isolated molecule in the above fragment. The molecular deformation electron density distribution is shown in Figure 3 of Peeters et al. [13].

1.2.1. Embedding of $B_{12}$ in Larger Graphenic Fragments. The largest Hartree-Fock computations were performed with 6 layers of $\mathrm{C}$ atoms surrounding the two $\mathrm{B}$ clusters shown in Figure 1. The geometry of $B_{12}$ in Figure $1(a)$ with 2 and $6 \mathrm{C}$ layers is very similar. For the cluster in part (b), the differences between the 2 and $6 \mathrm{C}$ layers are larger.

In general, of course, the relaxation of the $\mathrm{C}$ nuclei positions in the graphenic fragments on insertion of the $B_{12}$ impurity cluster is a function of the distance from the impurities. Table 2 in Peeters et al. records quantitative values of such relaxations. Grossly, the deviation $(y)$ seems to decay approximately as an inverse square root of the distance $(r)$ from the impurity: $y \approx c\left(1 / r^{1 / 2}\right)$ where $c$ is a constant. If one examines, though, the individual atomic deviations, a fair degree of scattering is in evidence, perhaps because of the outer edges of the graphenic nanofragment under discussion. The "zigzag" edges, such as those manifested in the study by Klein and Bytautus [19] give rise to "bond order" fluctuations near such edges. In [13], this was expected to be reflected as bond lengths fluctuate away from that of an infinite graphene monolayer.

We also note that the bonding electron density for the largest fragment considered by Peeters et al. [13] is shown in Figure 7 of their article. We want to conclude this subsection by emphasizing that in Section 2 below, quite a lot of attention is paid to the study of mechanical properties of graphenic fragments, that is, to observable manifestations of strains such as we have discussed microscopically above in this particular example of $B_{12}$ embedded in graphenic nanofragments.

\section{Nanotube Nanomechanics}

Since the discovery of carbon nanotubes (CNTs) by Iijima (1991) [20], we have witnessed an explosive development of the nanotube science and technology. There is a great variety of potential applications for nanotubes, ranging from energy storage, composites, nanoelectronics, and other solidstate devices, to sensors and actuators (see for example, the reviews of Baughman et al. [21] and Loiseau et al. [22]). 


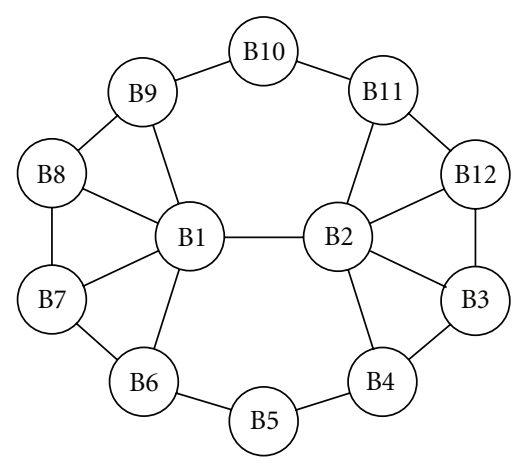

(a)

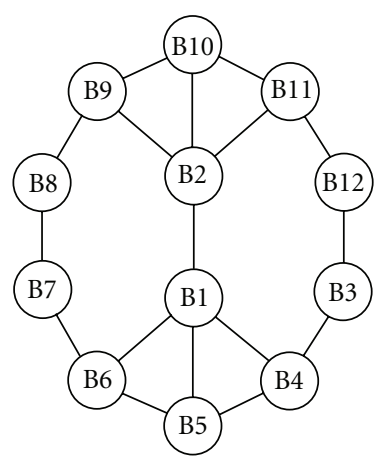

(b)

Figure 1: (a) Boron structure A embedded in graphenic fragments; (b) Boron structure B.

Several structural varieties of nanotubes have been identified and classified based on criteria such as helicity (also known as chirality), number of walls, and inclusion of pentagons-heptagons and. The simplest form is the singlewalled carbon nanotube (SWNT) which resembles a rolled honeycomb graphite layer into a mono-atomic-thick cylinder. Several concentrically embedded SWNTs form a multiwalled carbon nanotube (MWNT). Other nanotube varieties include nanotube bundles or ropes, intertube junctions, nanotori, coiled nanotubes, and so forth. Due to their relative simplicity and atomically precise morphology, single-walled carbon nanotubes offer the opportunity of assessing the validity of different macro- and microscopic models.

The properties of carbon nanotubes can be usefully grouped into three categories: structural, mechanical, and electronic. From the structural point of view, in most situations CNTs can be considered one-dimensional (1D) objects, with typical diameters $(\mathrm{dt})$ in the nm-range and lengths (L) reaching several micrometers. This one-dimensionality of tubes impacts on and is visible mostly through the mechanical and electronic properties. However, the structure of nanotubes can be exploited in itself such as for instance by field emitters or gas break-down sensors, which are based on the "sharpness" of CNTs giving rise to huge local electric fields.

While the prediction of electronic properties of carbon nanotubes required relatively subtle theoretical analysis, their unique mechanical behavior could be intuitively anticipated based on several features: strength of carbon bonds, their uniform arrangement within the graphitic sheet, and the seamless folding of this network into a tubule. The mechanical properties class is encompassing the elastic, thermal, vibrational, or any other properties related to the motion of the tube's atoms. In nanotubes, carbon is $\mathrm{sp}^{2}$-hybridized resulting in strong s-bonds weakly reinforced by $\pi$-bonds. Considering the hybridization, it is natural to assume a certain overlap between nanotube and graphite (graphene) elastic properties, such as Young's modulus, bending, tensile and torsional stiffness, and yield strength. SWNTs have tensile moduli close to $1 \mathrm{TPa}$ (stiff as diamond) and strengths $\approx 50 \mathrm{GPa}$ (corresponding to 5\%-10\% maximal strain), which earned them the title of ultimate fibers. Despite their stiffness, CNTs retain a high bending flexibility due to their high aspect ratios. With some exceptions, the thermal and vibrational properties of nanotubes also show similarities with graphite. Since the in-plane thermal conductivity of pyrolytic graphite is very high, it is to be expected that the on-axis thermal conductivity of defect-free tubes would be even higher.

The understanding of the mechanical response of nanotubes to external forces is of relevance for the application of nanotubes as a composite material reinforcement as well as in electronic devices, where the deformation of the tubes induced by the substrate alter locally the electronic properties of the nanotube. A broad discussion of potential applications of the nanotube can be found in existing reviews [23-26], also outlining the challenges of implementation. There are two actual applications already, where the carbon nanotube can commercially compete with other materials units, and the mechanics of carbon nanotubes plays either a central or an important secondary role in both cases.

Carbon as well as composite $\mathrm{BN}$ nanotubes demonstrate very high stiffness to an axial load or a bending of small amplitude, which translates in the record-high efficient linear-elastic moduli. At larger strains, the nanotubes (especially, the singlewalled type) are prone to buckling, kink forming, and collapse, due to the hollow shell-like structure. These abrupt changes (bifurcations) manifest themselves as singularities in the nonlinear stress-strain curve, but are reversible and involve no bond-breaking or atomic rearrangements. This resilience corresponds, quantitatively, to a very small subangstrom efficient thickness of the constituent graphitic shells. Irreversible yield of nanotubes begins at extremely high deformation (from several to dozens percent of in-plane strain, depending on the strain rate) and high temperature. The failure threshold (yield strength) turns out to depend explicitly on nanotube helicity, which thus demonstrates again the profound role of symmetry for the physical properties, either electrical conductivity or mechanical strength. Finally, the manifestation of mechanical strength in the multiwalled or bundled nanotubes (ropes) is obscured by the poor load transfer from the exterior to the core of such larger structures. 


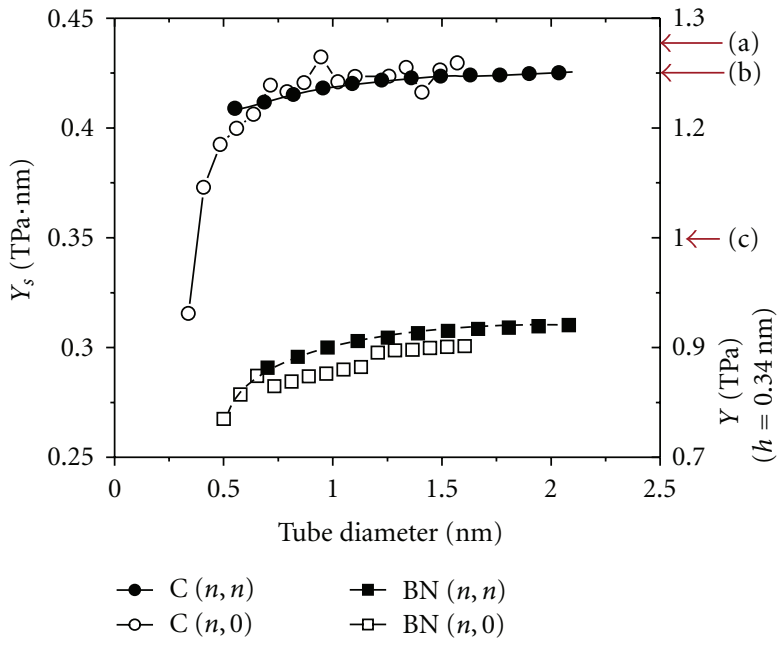

(a)

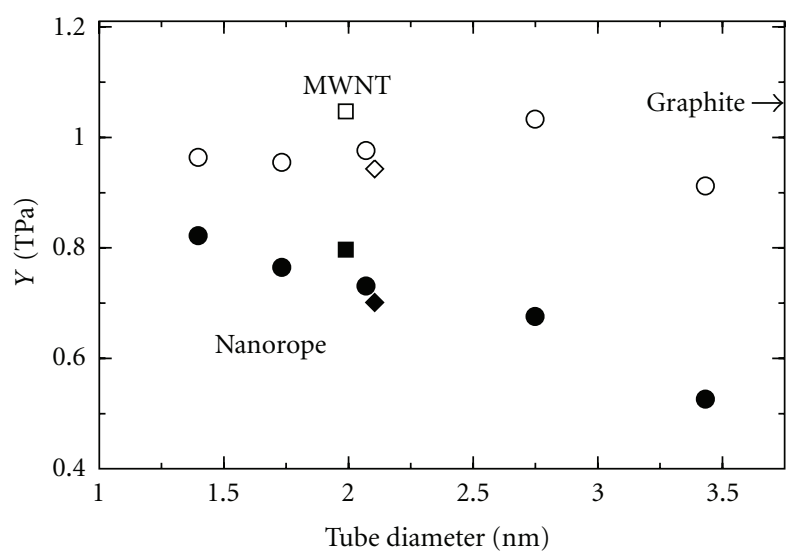

(b)

FIGURE 2: (a): Young modulus for armchair and zig-zag carbon and BN nanotubes. The values are given in the proper unit of TPa nm for SWNTs (left axis) and converted to TPa (right axis) by taking a value for the graphene thickness of $0.34 \mathrm{~nm}$. The experimental values for carbon nanotubes are reported on the right-hand side: (a) $1.28 \mathrm{TPa}$ [27]; (b) $1.25 \mathrm{TPa}$ [28]; (c) $1 \mathrm{TPa}$ for MWNTs [29]. (b): Young modulus versus tube diameter in different arrangements. Open symbols correspond to the multiwall geometry (10-layer tube with an interlayer distance of $0.34 \mathrm{~nm}$ ), and solid symbols for the single-wall-nanotube crystalline-rope configuration. In the MWNT geometry, the value of the Young modulus does not depend on the specific number of layers. The experimental value of the $c_{11}=1.06 \mathrm{TPa}$ elastic constant of graphite is also shown.

The similarities among graphite and other $\mathrm{sp}^{2}$-like bonded materials such as hexagonal boron nitride and boron-carbon-nitrogen compounds lead us to the theoretical proposition that $\mathrm{B}_{x} \mathrm{C}_{y} \mathrm{~N}_{z}$ nanotubes would be stable [3033]. Specific synthesis of these nanotubes was achieved afterwards: boron-nitride $[34,35]$ and $\mathrm{BC}_{2} \mathrm{~N}$ and $\mathrm{BC}_{3}[36$, 37] as well as other inorganic tubular forms of $\mathrm{WS}_{2}$ and $\mathrm{MoS}_{2}[38,39]$. The predicted properties of these tubules are quite different from those of carbon with numerous possible technological applications in the fields of catalysis, lubrication, electronic, and photonic devices [32, 33].

Calculations of the stiffness of SWNTs demonstrated that the Young modulus shows a small dependence on the tube diameter and chirality for the experimental range of nanotube diameters (between 1.3 and $1.4 \mathrm{~nm}$ ). It is predicted that carbon nanotubes have the highest Young's modulus of all the different types of composite tubes considered (BN, $\mathrm{BC}_{3}, \mathrm{BC}_{2} \mathrm{~N}, \mathrm{C}_{3} \mathrm{~N}_{4}, \mathrm{CN}$ ) [40]. Those results for the $\mathrm{C}$ and $\mathrm{BN}$ nanotubes are reproduced in the left panel of Figure 2. Furthermore, the Young modulus approaches, from below, the graphitic limit for diameters of the order of $1.2 \mathrm{~nm}$. The computed value of $\mathrm{C}$ for the wider carbon nanotubes of $0.43 \mathrm{TPa} \mathrm{nm}$, that corresponds to $1.26 \mathrm{TPa}$ modulus in our convention, is in excellent agreement with the experimental value for SWNT's of $1.25 \mathrm{TPa}$ [41]. It is also in rather good agreement with the value of $1.28 \mathrm{TPa}$ reported for multiwall nanotubes (MWNT) [27]. Although this result is for MWNT, the similarity between SWNT is not surprising as the intrawall $\mathrm{C}-\mathrm{C}$ bonds mainly determine the Young's modulus. From this result, we can estimate the Young modulus considering two different geometries of practical relevance:

(i) multiwall like geometry, in which the normal area is calculated using the wall-wall distance as the one in MWNTs, which is very approximately equal to the one of graphite,

(ii) nanorope or bundle configuration of SWNTs, where the tubes would be arranged forming a hexagonal close-packed lattice, with a lattice constant of $(2 R+$ 3.4), $R$ being the tube radius.

The results for these two cases are presented in the right panel of Figure 2. The MWNT geometry gives a value that is very close to the graphitic one; however, the rope geometry shows a decrease of the Young modulus with increasing the tube radius due to the quadratic increase of the effective area in this configuration, while the number of atoms increases only linearly with the tube diameter. The computed values for the SWNT ropes experimentally observed are, however, still very high $(0.5 \mathrm{TPa})$, even comparing with other known carbon fibers. This value is in quite good agreement with AFM experiments on anchored SWNTs ropes [42] ( $Y \sim$ $0.6 \mathrm{TPa}$ ) and for stress-strain puller measurements of the Young modulus for aligned nanotube ropes of MWNTs ( $Y$ $0.45 \pm 0.23 \mathrm{Tpa})[43]$.

For composite nanotubes, the results of [44] for the Young modulus of BN MWNT's give 1.22 TPa, which is somewhat larger than the result obtained for these tubes in the TB calculations $(\sim 0.9 \mathrm{TPa})$ [45], but nevertheless the agreement is close. We indicate that $\mathrm{BN}$ and $\mathrm{BC}_{3}$ tubes have similar values of the Young modulus (the calculated average 
Young modulus is $0.9 \mathrm{TPa}$ and $0.92 \mathrm{Tpa}$ for $\mathrm{BN}$ and $\mathrm{BC}_{3}$, resp.), although the latter have slightly larger values. In those studies $\mathrm{C}_{3} \mathrm{~N}_{4}$ nanotubes are shown to be much softer than any other type of tube, the reason being that for a given amount of tube surface, there is a smaller density of chemical bonds [40].

The Poisson ratio is given by the variation of the radius of the SWNT resulting from longitudinal deformations along the tube axis. In all cases, the computed Poisson ratio is positive: an elongation of the tube reduces its diameter. The $a b$ initio values are 0.14 (from 0.12 to 0.16 ) for the armchair $(n, n)$ tubes, and a little larger for other chiralities: 0.19 for $(10,0)$ and 0.18 for $(8,4)$. The uncertainty of the obtained values is of the order of $10 \%$. In summary, the ab initio calculations indicate that the Poisson ratio retains graphitic values except for a possible slight reduction for small radii. It shows chirality dependence: $(n, n)$ tubes display smaller values than $(10,0)$ and $(8,4)$. Similar differences are found between the $a b$ initio and TB calculations for $\mathrm{BN}$ tubes, namely, for the $(6,6)$ tube the ab-initio value for the Poisson ratio is 0.23 whereas the $\mathrm{TB}$ one is 0.30 [45].

2.1. Experimental Evidence of Nanotube Resilience. Collapsed forms of the nanotube ("nanoribbons") have been observed in experiment, and their stability can be explained by the competition between the van der Waals attraction and elastic energy. The basic physics can be understood by noticing that the elastic curvature energy per unit length is proportional to $1 / R$ ( $R$, radii of the tube); however, for a fully collapsed single-wall tubule with the opposite tubule walls at the typical van der Waals contact distance $c$, the energy per unit length would be composed of a higher curvature energy due to the edges which is independent of the initial tubule diameter, and a negative van der Waals contribution, $\mathcal{E}_{\mathrm{vdW}}$ 0.03-0.04 eV/atom, that is, $\propto R$ per unit length. Collapse occurs when the latter term prevails above a certain critical tube radii $R_{c}$ that depends on the number $N$ of shells of the nanotube, $R_{c}(N=1) \sim 8 c$ and $R_{c}(N=8) \sim 19 c$ [46], and the thickness of the collapsed strip-ribbon is $(2 \mathrm{~N}$ 1)c. Any additional torsional strain imposed on a tube in experimental environment also favors flattening $[47,48]$ and facilitates the collapse.

The bending seems fully reversible up to very large bending angles despite the occurrence of kinks and highly strained tubule regions in simulations, that are in excellent morphological agreement with the experimental images [49]. Similar bent-buckled shapes have been reported by several groups [49-51], Figure 4.4. This apparent flexibility stems from the ability of the $\mathrm{sp}^{2}$ network to rehybridize when deformed out of plane, with the degree of $\mathrm{sp}^{2}-\mathrm{sp}^{3}$ rehybridization being proportional to the local curvature [52].

In this context, the recent work of Colombo [53, 54] is very relevant as it presents a very detailed combined continuum and atomistic approach to the elastic properties of graphene and $\mathrm{C}$ nanoribbons. In a little more detail, the stress-strain nonlinear constitutive equation is worked out by Colombo using continuum theory in both small-strain and large-strain circumstances. Then by atomistic simulations, the relevant linear and non-linear elastic moduli have been computed (see also, [53]). In particular, Colombo throws light on the physical meaning of the effective non-linear elastic modulus measured by nanoindentation of a freestanding monolayer graphene sample [55]. Through a stressstrain plot, Colombo also predicts a very high failure stress, in good accordance with experimental data recorded by Lee et al. [55]. The corresponding effective 3D failure stress is found by Colombo to be $\sim 1300 \mathrm{~Pa}$, which exceeds that in most materials, including multiwalled nanotubes.

In relation to bending elasticity, Colombo identifies the bending rigidity modulus by continuum theory and characterizes it by the local curvature of a bended ribbon. Then this bending modulus is calculated by tight-binding atomistic simulations (see also [54]). Finally, the elastic behaviour of various curved nanoribbons is studied and the onset of nanoscale features is discussed. It is shown, in particular, that atomic-scale relaxations on bending induce an additional strain field of in-plane stretching. An important issue is then raised as to the way in which to disentangle bending and stretching features for small-width nanoribbons.

\section{Parallels between Low-Dimensional Assemblies of $\mathrm{C}$ and of $\mathrm{Si}$ and Ge Atoms}

Our interest in this area has been rekindled by the very recent study of Cahangirov et al. [56]. These authors reported calculations of structure optimization, phonon modes, and finite temperature molecular dynamics. Their predictions were that both $\mathrm{Si}$ and $\mathrm{Ge}$ can have stable, two-dimensional, low-buckled honeycomb structures. Therefore, in this paper, we shall, first of all, use available work from both experiment and theory to compare especially $\mathrm{Si}-\mathrm{Si}$ equilibrium bond lengths in a number of structures, both finite molecular and clusters and in honeycomb lattices, with the geometry predicted in [6] for $\mathrm{Si}_{6} \mathrm{H}_{6}$. It is natural here, therefore, to elaborate somewhat on the results reported in [6].

First of all, results were obtained on the equilibrium geometry of $\mathrm{Si}_{6} \mathrm{H}_{6}$ from spin-compensated (restricted) HF (HRF) calculations. The bond lengths predicted by this method were $2.21 \AA$ for the Si-Si bonds and for the Si-H lengths were $1.47 \AA$. Also binding was demonstrated with respect to $6 \mathrm{Si}$ and $6 \mathrm{H}$ isolated atoms.

But a central part of the study in [6] was to consider the stretching of chemical bonds in the (as yet unsynthesized) $\mathrm{Si}_{6} \mathrm{H}_{6}$ molecule. Such stretching was first carried out by Grassi et al. [6] in which the stretching was uniform; that is, all $\mathrm{Si}-\mathrm{Si}$ and $\mathrm{Si}-\mathrm{H}$ bonds were changed by a common scale factor $\lambda$. Figure 1 in [6] displays before RHF and UHF potential energy curves of $\mathrm{Si}_{6} \mathrm{H}_{6}$ as a function of $\lambda$.

3.1. Itinerant versus Localized $\pi$-Electrons. Since there is a sense in which a UHF treatment reflects electron "correlation" by putting $\uparrow$ and $\downarrow$ spins in different space orbitals, Grassi et al. [6] attempted to draw conclusions about itinerant versus localized behaviour of $\pi$-electrons in $\mathrm{Si}_{6} \mathrm{H}_{6}$. Of course, the "sister" molecule benzene is a form for comparison. In this latter system, the diamagnetic 
TABle 1: Bond-lengths for different Si-structures $(\AA)^{*}$. Note that for the $\mathrm{Si}=\mathrm{Si}$ double bond lengths of disilenes vary between 2.14 and $2.29 \AA$ and are nearly 5 to $10 \%$ shorter than the Si-Si single bond lengths of corresponding disilanes. This rate of bond shortening is less than $13 \%$ in carbon compounds [57].

\begin{tabular}{lc}
\hline Si-bulk [59] & 2.35 \\
\hline $\mathrm{Si}_{2}$ (en silicon-carbene-complex) [60] & $2.2294 \pm 0.0011$ \\
Si-nanowire (H-terminated) [57] & 2.34 to 2.36 \\
nanocrystal: $\mathrm{Si}_{87} \mathrm{H}_{76}[57]$ & 2.35 \\
\hline $\mathrm{Si}_{6} \mathrm{H}_{6} \mathrm{Si}-\mathrm{H}[6]^{*}$ & 1.47 \\
\hline${ }^{*}$ Compare 1.07 $\AA$ for C-H length in benzene, which is the same as in the $\mathrm{CH}$ \\
radical itself.
\end{tabular}

susceptibility plus properties relating to ring currents testify, from experiment, to the delocalization of the $\pi$-electrons. In their Figures 3 and 4, Grassi et al. [6] display the spin population magnitudes on the $\mathrm{Si}$ and $\mathrm{H}$ atoms of the $\mathrm{Si}_{6} \mathrm{H}_{6}$ ring, as a function of uniform stretching $\lambda$. Their conclusion was that the $\pi$-electrons have their densities dominantly around the Si nuclei, with a tendency towards delocalization (ring currents) for low values of the scaling factor $\lambda$.

As already mentioned in Section 1.1 above, in [6] it was demonstrated that for planar $\mathrm{Si}_{6} \mathrm{H}_{6}$ a study of the normal modes of vibration reveals that not all the vibrational frequencies are real. This led these authors to consider the effect of buckling of the planar structure on these frequencies. The minimum energy structure was obtained by an optimization procedure at the UHF-singlet level. The buckled structure was such that all vibrational mode frequencies were now real. The reader is referred to [6] if fuller details are eventually required.

To begin the above programme, we collect in Table 1 available results, which are mainly from presently available theoretical and experimental work, for Si-Si equilibrium bond lengths in some rather different environments. Thus, for the predicted buckled $\mathrm{Si}_{6} \mathrm{H}_{6}$ molecule in [6], the restricted HF prediction is $2.21 \AA$ (see Table 1 of [6]). One experimentally accessible $\mathrm{Si}-\mathrm{Si}$ distance is from a study of an Si nanowire [57] and from gas-phase infrared spectra of cationic Si-clusters $(n=6-17)$; see supplementary material in Reference [58]. Returning to theory, we next note that Si nanowires potentially have structures resembling assemblies of smaller Si clusters.

In this context, it is relevant to mention work on the silicon clathrate compound $(\mathrm{Na}, \mathrm{Ba})_{X} \mathrm{Si}_{46}$ studied in Kawaji et al. [61]. As these authors stress, the Si-Si atoms are bonded tetrahedrally with approximately the same bond distances as in ordinary diamond structure of $\mathrm{Si}$. These distances are recorded in Table 1. We have also added Si-Si lengths in some smaller Si free space clusters (see, e.g., Alonso's book [62]). It is also relevant to refer to work of Phillips [63] at this point. He concluded from analyzing fragmentation and contration experiments that Si clusters with less that ten atoms have a unique structure with little or no resemblance to the diamond lattice. It is of interest to refer to Figure 9 of Merson and Sattler [64] which depicts electron density isosurfaces of the fullerenic $\mathrm{Si}_{24}$. The left-side of their Figure
9 gives a side view with a pentagonal-like surface. The righthand side shows a hexagonal of Si atoms.

At this point, we return to the work of Cahangirov et al. [56]. In their figures, these authors first present the calculated variations of the binding energy of the relaxed honeycomb structure of $\mathrm{Si}$ and $\mathrm{Ge}$ as a function of lattice constant. In that Figure, planar (PL), low-buckled (LB), and highbuckled (HB) honeycomb structures correspond to distinct minima. They find that the PL honeycomb structure is the least energetic state and is not stable. They then address the issue as to whether these puckered LB and $\mathrm{HB}$ geometries correspond to real local minima in the Born-Oppenheimer surface.

The PL structure of Si has some phonon modes which have imaginary frequencies analogous to planar $\mathrm{Si}_{6} \mathrm{H}_{6}$ reported by Grassi et al. [6].

In contrast, the phonon dispersion curves shown in Figure 1 of Cahangirov et al. [56] indicate that the 2D periodic LB honeycomb structure of $\mathrm{Si}$ is stable. There is an equilibrium buckling of $0.44 \AA$ for $\mathrm{Si}$ and $0.64 \AA$ for Ge. The stability of these LB structures of both $\mathrm{Si}$ and Ge was tested in addition by the means of extensive ab initio finite temperature molecular dynamics calculations using time steps of $2 \times 10^{-15}$ secs. They record the facts that the 2D periodic $\mathrm{LB}$ structure of $\mathrm{Si}(\mathrm{Ge})$ is not lost by raising the temperature from $T=0$ to $1000 \mathrm{~K}(800 \mathrm{~K})$ for 10 picoseconds (ps). In this paper, it is especially noteworthy that a finite size, large hexagonal LB flake of $\mathrm{Si}(\mathrm{Ge})$, with hydrogen passivated edge atoms, remains intact on raising $T$ from 0 to $1000 \mathrm{~K}$ $(800 \mathrm{~K})$ in 100 steps, and holding it there for more than 3 ps.

Finally, we note that calculated band structures and their corresponding electronic density of states of LB Si and $\mathrm{Ge}$ are presented in Figure 2 of Cahangirov et al. [56]. Also their Table 1 summarizes binding energy and structural parameters calculated for the LB honeycomb structure. As to possible subsequent experiments on the synthesis of $\mathrm{Si}$ and Ge nanoribbons with honeycomb structures, it is of interest to add that recent work by Nakano et al. [65] reports the soft synthesis of a single Si monolayer sheet on a substrate.

\section{Future Directions}

Superconducting graphene has recently been predicted [6668] with a high transition temperature, though at the time of writing no experimental discovery has been reported. The discussion of the possible pairing mechanism in graphite intercalated compounds (GIC) by Capone et al. [69] is also relevant in the present context (see for example, the recent work by Pellegrino et al. [70] on relevant topic of pairing symmetry). However, there is clearly an area of potential interest there, both fundamental and technological, for the future.

Also, in connection with low-dimensional C-atom structures, we want to mention work on experimental detection of plasmon modes which has been utilized to investigate the dynamical behaviour of electrons in graphene layers [71-74]. In this general area, it is also relevant here to note the very recent study of Wang and Chakraborty [75] 
on Coulomb screening and collective excitations in biased bilayer graphene. The plasmon-phonon strongly coupled mode in doped graphene has been explored by Liu and Willis [76].

Additionally, in relation to our discussion of strain effects above, the study of Choi et al. [77] has reported calculations on electronic properties of graphene subjected to uniaxial and isotropic strains, respectively. As consequences of these numerical studies, the semimetallic character is predicted to persist up to a very large uniaxial strain of some $30 \%$, except in a very narrow strain range where a tiny energy gap is predicted. The work function is studied theoretically also, and is found to increase substantially as both the uniaxial and the isotropic strains increase. The impact on the optical conductivity has been addressed by Pellegrino et al. [78, 79] .

Of potential fundamental interest for the future, we note the very recent theoretical work by Novoselov et al. [80] on the theory of the quantum Hall effect (QHE) in finite graphene devices. Already, prior to this study, several experiments have examined the QHE in graphene [81-83]. In the theoretical work of Novoselov et al. [80], the QHE in graphene is studied based on the current injection model, which takes some account of the finite rectangular geometry with source and drain electrodes. In their work, the presence of disorder does not play a significant role. Instead, the boundary conditions during the injection into the graphene sheet, which are enforced by the presence of the Ohmic contacts, determine the current-voltage characteristics.

\section{Acknowledgments}

The collaboration with many people over the years has made possible the results presented in this paper. In particular, N.H. March wishes to thank Dr. A. Peeters, professors G.G.N. Angilella, C. Van Alsenoy, I.A. Howard, and D.J. Klein for invaluable cooperation on the general area embraced by this paper. Also A. Rubio wants to thank M. Cohen, S.G. Louie, P.M. Ajayan, A. Zettl, Y. Miyamoto, P. Bernier, and C. Goze for their fundamental contributions to the results presented here. This work has been supported also by the Spanish MEC (FIS2007-65702-C02-01), ACI-Promociona (ACI2009-1036), "Grupos Consolidados UPV/EHU del Gobierno Vasco" (IT-319-07), ETORTEK and the European Union through e-I3 ETSF (Contract: 211956), NANANODEVICES (IST-2006-029192), and THEMA (Contract: 228539) projects. The authors acknowledge support by the Barcelona Supercomputing Center, "Red Española de Supercomputacion”, SGIker ARINA (UPV/EHU), Transnational Access Programme HPC-Europe++. Finally, it is a pleasure for N. H. March to thank professor P. M. Echenique for most generous hospitality at DIPC.

\section{References}

[1] N. H. March, "Theoretical determination of the electron distribution in Benzene by the Thomas-Fermi and the molecularorbital methods," Acta Crystallographica, vol. 5, pp. 187-193, 1952.
[2] N. H. March, Self-Consistent Fields in Atoms, Elsevier, Amsterdam, The Netherlands, 1975.

[3] R. G. Parr and W. Yang, Density-Functional Theory of Atoms and Molecules, Oxford University Press, Oxford, UK, 1989.

[4] S. C. Abrahams, J. M. Robertson, and J. G. White, "The crystal and molecular structure of naphthalene. I. X-ray measurements," Acta Crystallographica, vol. 2, pp. 233-238, 1949.

[5] S. C. Abrahams, J. M. Robertson, and J. G. White, "The crystal and molecular structure of naphthalene. II. Structure investigation by the triple Fourier series method," Acta Crystallographica, vol. 2, pp. 238-244, 1949.

[6] A. Grassi, G. M. Lombardo, R. Pucci, G. G. N. Angilella, F. Bartha, and N. H. March, "Stretched chemical bonds in $\mathrm{Si}_{6} \mathrm{H}_{6}$ : a transition from ring currents to localized $\pi$-electrons?" Chemical Physics, vol. 297, no. 1-3, pp. 13-19, 2004.

[7] C. A. Coulson and I. Fischer, "Notes on the molecular-orbital treatment of the hydrogen molecule," Philosophical Magazine, vol. 40, pp. 386-393, 1949.

[8] Y. Wang, C. Sun, J. Zou et al., "Oxygen vacancy induced structural variations of exfoliated monolayer $\mathrm{MnO}_{2}$ sheets," Physical Review B, vol. 81, no. 8, Article ID 081401, 4 pages, 2010.

[9] J. C. Meyer, A. K. Geim, M. I. Katsnelson, K. S. Novoselov, T. J. Booth, and S. Roth, "The structure of suspended graphene sheets," Nature, vol. 446, no. 7131, pp. 60-63, 2007.

[10] S. Stankovich, D. A. Dikin, G. H. B. Dommett et al., "Graphene-based composite materials," Nature, vol. 442, no. 7100, pp. 282-286, 2006.

[11] D. Moran, A. C. Simmonett, F. E. Leach III, W. D. Allen, P. V. R. Schleyer, and H. F. Schaefer III, "Popular theoretical methods predict benzene and arenes to be nonplanar," Journal of the American Chemical Society, vol. 128, no. 29, pp. 9342-9343, 2006.

[12] F. Dietz, N. Tyutyulkov, G. Madjarova, and K. Müllen, "Is 2-D Graphite an Ultimate Large Hydrocarbon? II. Structure and Energy Spectra of Polycyclic Aromatic Hydrocarbons with Defects," Journal of Physical Chemistry B, vol. 104, no. 8, pp. 1746-1761, 2000.

[13] A. Peeters, C. Van Alsenoy, N. H. March, D. J. Klein, and V. E. Van Doren, "Boron $\mathrm{B}_{12}$ cluster embedded in graphitic fragments," Journal of Physical Chemistry B, vol. 105, no. 43, pp. 10546-10553, 2001.

[14] H.-Y. Zhu, D. J. Klein, W. A. Seitz, and N. H. March, "BN alternants: boron nitride cages and polymers," Inorganic Chemistry, vol. 34, no. 6, pp. 1377-1383, 1995.

[15] H.-Y. Zhu, D. J. Klein, N. H. March, and A. Rubio, "Small band-gap graphitic CBN layers," Journal of Physics and Chemistry of Solids, vol. 59, no. 8, pp. 1303-1308, 1998.

[16] A. Stabel, P. Herwig, K. Müllen, and J. P. Rabe, "Diodelike current-voltage curves for a single molecule-tunneling spectroscopy with submolecular resolution of an alkylated, peri-condensed hexabenzocoronene," Angewandte Chemie, vol. 34, no. 15, pp. 1609-1611, 1995.

[17] I. Boustani, "Systematic ab initio investigation of bare boron clusters: determination of the geometry and electronic structures of Bn (n=2-14)," Physical Review B, vol. 55, no. 24, pp. 16426-16438, 1997.

[18] I. Boustani, "Structure and stability of small boron clusters. A density functional theoretical study," Chemical Physics Letters, vol. 240, no. 1-3, pp. 135-140, 1995.

[19] D. J. Klein and L. Bytautas, "Graphitic Edges and Unpaired $\pi$ Electron Spins," Journal of Physical Chemistry A, vol. 103, no. 26, pp. 5196-5210, 1999. 
[20] S. Iijima, "Helical microtubules of graphitic carbon," Nature, vol. 354, no. 6348, pp. 56-58, 1991.

[21] R. H. Baughman, A. A. Zakhidov, and W. A. De Heer, "Carbon nanotubes - the route toward applications," Science, vol. 297, no. 5582, pp. 787-792, 2002.

[22] A. Loiseau, P. Launois-Bernede, P. Petit, S. Roche, and J.-P. Salvetat, "Understanding carbon nanotubes," Lecture Notes in Physics, vol. 677, p. 555, 2006.

[23] T. W. Ebbesen, Carbon Nanotubes: Preparation and Properties, CRC Press, Boca Raton, Fla, USA, 1997.

[24] P. M. Ajayan and T. W. Ebbesen, "Nanometre-size tubes of carbon," Reports on Progress in Physics, vol. 60, no. 10, pp. 1025-1062, 1997.

[25] B. I. Yakobson and R. E. Smalley, "Fullerene nanotubes: $\mathrm{C}_{1,000,000}$ and beyond," American Scientist, vol. 85, no. 4, pp. 324-337, 1997.

[26] J. Bernholc, C. Roland, and B. I. Yakobson, "Nanotubes," Current Opinion in Solid State and Materials Science, vol. 2, no. 6, pp. 706-715, 1997.

[27] E. W. Wong, P. E. Sheehan, and C. M. Lieber, "Nanobeam mechanics: elasticity, strength, and toughness of nanorods and nanotubes," Science, vol. 277, no. 5334, pp. 1971-1975, 1997.

[28] A. Krishnan, E. Dujardin, T. W. Ebbesen, P. N. Yianilos, and M. M. J. Treacy, "Young's modulus of single-walled nanotubes," Physical Review B, vol. 58, no. 20, pp. 14013-14019, 1998.

[29] J. Muster, M. Burghard, S. Roth, G. S. Duesberg, E. Hernández, and A. Rubio, "Scanning force microscopy characterization of individual carbon nanotubes on electrode arrays," Journal of Vacuum Science and Technology B, vol. 16, no. 5, pp. 27962801, 1998.

[30] A. Rubio, J. L. Corkill, and M. L. Cohen, "Theory of graphitic boron nitride nanotubes," Physical Review B, vol. 49, no. 7, pp. 5081-5084, 1994.

[31] A. Rubio, "Nanocomposite tubules: A new class of materials from theory," Condensed Matter News, vol. 6, p. 6, 1997.

[32] Y. Miyamoto, A. Rubio, M. L. Cohen, and S. G. Louie, "Chiral tubules of hexagonal $\mathrm{BC}_{2} \mathrm{~N}$," Physical Review B, vol. 50, no. 7, pp. 4976-4979, 1994.

[33] Y. Miyamoto, A. Rubio, S. G. Louie, and M. L. Cohen, "Electronic properties of tubule forms of hexagonal $\mathrm{BC}_{3}$," Physical Review B, vol. 50, no. 24, pp. 18360-18366, 1994.

[34] N. G. Chopra, R. J. Luyken, K. Cherrey et al., "Boron nitride nanotubes," Science, vol. 269, no. 5226, pp. 966-967, 1995.

[35] A. Loiseau, F. Willaime, N. Demoncy, G. Hug, and H. Pascard, "Boron nitride nanotubes with reduced numbers of layers synthesized by arc discharge," Physical Review Letters, vol. 76, no. 25, pp. 4737-4740, 1996.

[36] Z. Weng-Sieh, K. Cherrey, N. G. Chopra et al., "Synthesis of $\mathrm{B}_{x} \mathrm{C}_{y} \mathrm{~N}_{z}$ nanotubules," Physical Review B, vol. 51, no. 16, pp. 11229-11232, 1995.

[37] O. Stephan, P. M. Ajayan, C. Colliex et al., "Doping graphitic and carbon nanotube structures with boron and nitrogen," Science, vol. 266, no. 5191, pp. 1683-1685, 1994.

[38] R. Tenne, L. Margulis, M. Genut, and G. Hodes, "Polyhedral and cylindrical structures of tungsten disulphide," Nature, vol. 360, pp. 444-446, 1992.

[39] R. Tenne, "Doped and heteroatom-containing fullerene-like structures and nanotubes," Advanced Materials, vol. 7, pp. 965-995, 1995.

[40] E. Hernández, C. Goze, P. Bernier, and A. Rubio, "Elastic properties of single-wall nanotubes," Applied Physics A, vol. 68, no. 3, pp. 287-292, 1999.
[41] M. M. J. Treacy, T. W. Ebbesen, and J. M. Gibson, "Exceptionally high Young's modulus observed for individual carbon nanotubes," Nature, vol. 381, no. 6584, pp. 678-680, 1996.

[42] J.-P. Salvetat, G. A. D. Briggs, J.-M. Bonard et al., "Elastic and shear moduli of single-walled carbon nanotube ropes," Physical Review Letters, vol. 82, no. 5, pp. 944-947, 1999.

[43] Z. W. Pan, S. S. Xie, L. Lu et al., "Tensile tests of ropes of very long aligned multiwall carbon nanotubes," Applied Physics Letters, vol. 74, no. 21, pp. 3152-3154, 1999.

[44] N. G. Chopra and A. Zettl, "Measurement of the elastic modulus of a multi-wall boron nitride nanotube," Solid State Communications, vol. 105, no. 5, pp. 297-300, 1998.

[45] E. Hernández, C. Goze, P. Bernier, and A. Rubio, "Elastic properties of $\mathrm{C}$ and $\mathrm{B}_{x} \mathrm{C}_{y} \mathrm{~N}_{z}$ composite nanotubes," Physical Review Letters, vol. 80, no. 20, pp. 4502-4505, 1998.

[46] N. G. Chopra, L. X. Benedict, V. H. Crespi, M. L. Cohen, S. G. Louie, and A. Zettl, "Fully collapsed carbon nanotubes," Nature, vol. 377, no. 6545, pp. 135-138, 1995.

[47] B. I. Yakobson, C. J. Brabec, and J. Bernholc, "Nanomechanics of carbon tubes: instabilities beyond linear response," Physics Review Letters, vol. 76, pp. 2511-2514, 1996.

[48] B. I. Yakobson, C. J. Brabec, and J. Bernholc, "Structural mechanics of carbon nanotubes: from continuum elasticity to atomistic fracture," Journal of Computer-Aided Materials Design, vol. 3, no. 1-3, pp. 173-182, 1996.

[49] S. Iijima, C. Brabec, A. Maiti, and J. Bernholc, "Structural flexibility of carbon nanotubes," Journal of Chemical Physics, vol. 104, no. 5, pp. 2089-2092, 1996.

[50] J. F. Despres, E. Daguerre, and K. Lafdi, "Flexibility of graphene layers in carbon nanotubes," Carbon, vol. 33, no. 1, pp. 87-89, 1995.

[51] R. S. Ruoff and D. C. Lorents, Bulletin of the APS, vol. 40, p. 173, 1995.

[52] R. C. Haddon, "Chemistry of the fullerenes: the manifestation of strain in a class of continuous aromatic molecules," Science, vol. 261, no. 5128, pp. 1545-1550, 1993.

[53] E. Cadelano, P. L. Palla, S. Giordano, and L. Colombo, "Nonlinear elasticity of monolayer graphene," Physical Review Letters, vol. 102, no. 23, Article ID 235502, 2009.

[54] E. Cadelano, S. Giordano, and L. Colombo, "Elastic properties of graphene and carbon nanoribbons: a combined continuum and atomistic approach," submitted for publication.

[55] C. Lee, X. Wei, J. W. Kysar, and J. Hone, "Measurement of the elastic properties and intrinsic strength of monolayer graphene," Science, vol. 321, no. 5887, pp. 385-388, 2008.

[56] S. Cahangirov, M. Topsakal, E. Aktürk, H. Šahin, and S. Ciraci, "Two- and one-dimensional honeycomb structures of silicon and germanium," Physical Review Letters, vol. 102, no. 23, Article ID 236804, 2009.

[57] M. Palummo, F. Iori, R. Del Sole, and S. Ossicini, "Giant excitonic exchange splitting in Si nanowires: first-principles calculations," Physical Review B, vol. 81, no. 12, Article ID 121303, 4 pages, 2010.

[58] J. T. Lyon, P. Gruene, A. Fielicke et al., "Structures of silicon cluster cations in the gas phase," Journal of the American Chemical Society, vol. 131, no. 3, pp. 1115-1121, 2009.

[59] O. Madelung, Ed., Semiconductors_Basic Data, Springer, Berlin, Germany, 1996.

[60] Y. Wang, Y. Xie, P. Wei et al., "A stable silicon(0) compound with a $\mathrm{Si}=\mathrm{Si}$ double bond," Science, vol. 321, no. 5892, pp. 1069-1071, 2008. 
[61] H. Kawaji, H.-O. Horie, S. Yamanaka, and M. Ishikawa, "Superconductivity in the silicon clathrate compound ( $\mathrm{Na}$, $\mathrm{Ba})_{x} \mathrm{Si}_{46}$," Physical Review Letters, vol. 74, no. 8, pp. 1427-1429, 1995.

[62] J. A. Alonso, Structure And Properties of Atomic Nanoclusters, Imperial College Press, London, UK, 2006.

[63] J. C. Phillips, "Morphology of medium-size silicon clusters," The Journal of Chemical Physics, vol. 88, no. 3, pp. 2090-2091, 1988.

[64] B. Marsen and K. Sattler, "Fullerene-structured nanowires of silicon," Physical Review B, vol. 60, no. 16, pp. 11593-11600, 1999.

[65] H. Nakano, T. Mitsuoka, M. Harada et al., "Soft synthesis of single-crystal silicon monolayer sheets," Angewandte Chemie, vol. 45, no. 38, pp. 6303-6306, 2006.

[66] S. Pathak, V. B. Shenoy, and G. Baskaran, "Possible hightemperature superconducting state with a d+id pairing symmetry in doped graphene," Physical Review B, vol. 81, no. 8, Article ID 085431, 5 pages, 2010.

[67] A. M. Black-Schaffer and S. Doniach, "Resonating valence bonds and mean-field d-wave superconductivity in graphite," Physical Review B, vol. 75, no. 13, Article ID 134512, 2007.

[68] B. Uchoa and A. H. Castro Neto, "Superconducting states of pure and doped graphene," Physical Review Letters, vol. 98, no. 14, Article ID 146801, 2007.

[69] M. Capone, M. Fabrizio, C. Castellani, and E. Tosatti, "Colloquiumml: modeling the unconventional superconducting properties of expanded A3 C60 fullerides," Reviews of Modern Physics, vol. 81, no. 2, pp. 943-958, 2009.

[70] F. M.D. Pellegrino, G. G.N. Angilella, and R. Pucci, "Pairing symmetry of superconducting graphene," European Physical Journal B, vol. 76, no. 3, pp. 469-473, 2010.

[71] C. Kramberger, R. Hambach, C. Giorgetti et al., "Linear plasmon dispersion in single-wall carbon nanotubes and the collective excitation spectrum of graphene," Physical Review Letters, vol. 100, no. 19, Article ID 196803, 2008.

[72] Y. Liu, R. F. Willis, K. V. Emtsev, and TH. Seyller, "Plasmon dispersion and damping in electrically isolated twodimensional charge sheets," Physical Review B, vol. 78, no. 20, Article ID 201403, 2008.

[73] A. Bostwick, T. Ohta, T. Seyller, K. Horn, and E. Rotenberg, "Quasiparticle dynamics in graphene," Nature Physics, vol. 3, no. 1, pp. 36-40, 2007.

[74] X.-F. Wang and T. Chakraborty, "Collective excitations of Dirac electrons in a graphene layer with spin-orbit interactions," Physical Review B, vol. 75, no. 3, Article ID 033408, 2007.

[75] X. -F. Wang and T. Chakraborty, "Coulomb screening and collective excitations in biased bilayer graphene," Physical Review B, vol. 81, no. 8, Article ID 081402, 4 pages, 2010.

[76] Y. Liu and R. F. Willis, "Plasmon-phonon strongly coupled mode in epitaxial graphene," Physical Review B, vol. 81, no. 8, Article ID 081406, 4 pages, 2010.

[77] S. -M. Choi, S. -H. Jhi, and Y. -W. Son, "Effects of strain on electronic properties of graphene," Physical Review B, vol. 81, no. 8, Article ID 081407, 4 pages, 2010.

[78] F. M. D. Pellegrino, G. G. N. Angilella, and R. Pucci, "Strain effect on the optical conductivity of graphene," Physical Review B, vol. 81, no. 3, Article ID 035411, 12 pages, 2010.

[79] T. Kramer, C. Kreisbeck, V. Krueckl, E. J. Heller, R. E. Parrott, and C. T. Liang, "Theory of the quantum Hall effect in finite graphene devices," Physical Review B, vol. 81, Article ID 081410, 4 pages, 2010.
[80] K. S. Novoselov, A. K. Geim, S. V. Morozov et al., "Twodimensional gas of massless Dirac fermions in graphene," Nature, vol. 438, no. 7065, pp. 197-200, 2005.

[81] K. S. Novoselov, A. K. Geim, S. V. Morozov et al., "Twodimensional gas of massless Dirac fermions in graphene," Nature, vol. 438, no. 7065, pp. 197-200, 2005.

[82] Y. Zhang, Y.-W. Tan, H. L. Stormer, and P. Kim, "Experimental observation of the quantum Hall effect and Berry's phase in graphene," Nature, vol. 438, no. 7065, pp. 201-204, 2005.

[83] Y. Zhang, Z. Jiang, J. P. Small et al., "Landau-level splitting in graphene in high magnetic fields," Physical Review Letters, vol. 96, no. 13, Article ID 136806, pp. 1-4, 2006. 

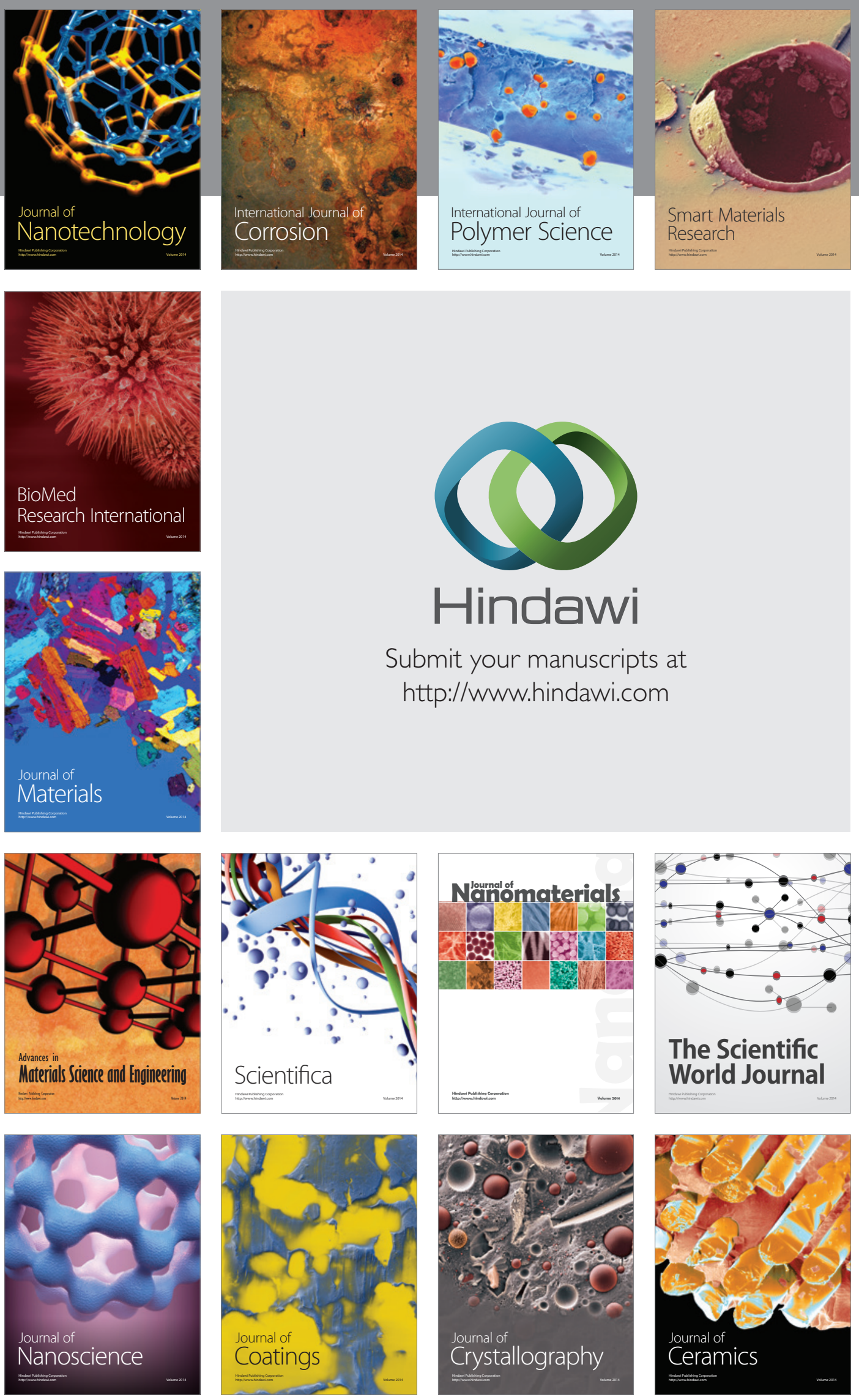

The Scientific World Journal

Submit your manuscripts at

http://www.hindawi.com

\section{World Journal}

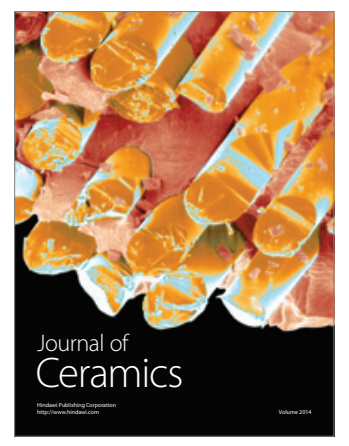

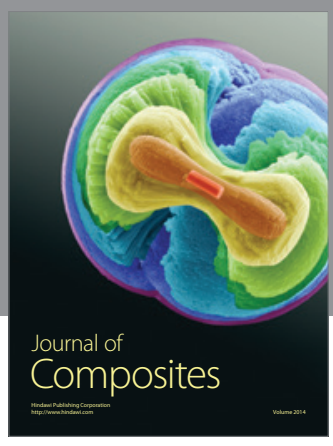
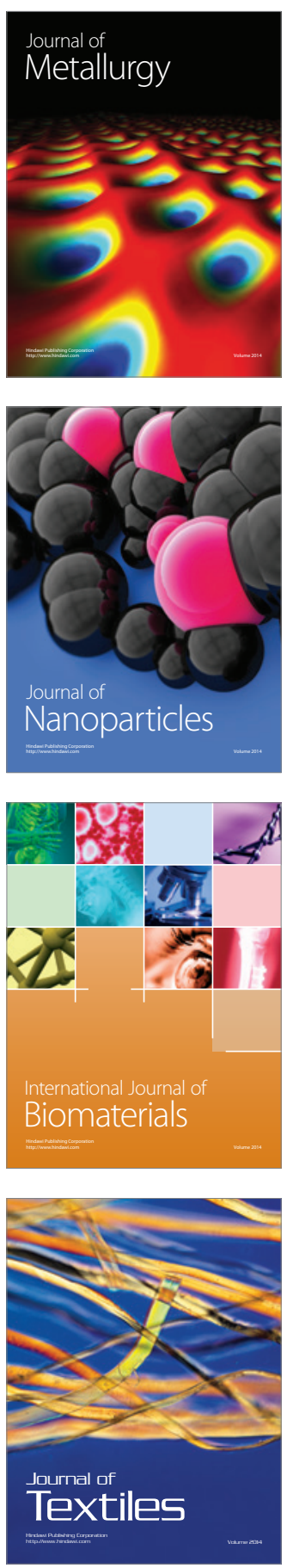